\title{
ELECTRON-PHONON DRIVEN SPIN FRUSTRATION IN MULTI-BAND HUBBARD MODELS: MX CHAINS AND OXIDE SUPERCONDUCTORS
}

\author{
J. Tinka GAMmeL, ${ }^{(a)}$ K. YOnEMitSu, ${ }^{(b)}$ A. SAXENA, ${ }^{(b)}$ A.R. Bishop, ${ }^{(b)}$ and H. Röder ${ }^{(c)}$ \\ (a) Code 573, Materials Research Branch, NCCOSC RDT\&E Division (NRaD), San Diego, CA 92152- \\ 5000, USA \\ (b) Theoretical Division and Centers for Nonlinear Studies and Materials Science, Los Alamos \\ National Laboratory, Los Alamos, New Mexico 87545, USA \\ (c) Physikalishes Institüt, Universität Bayreuth, W-8580 Bayreuth, Germany
}

\begin{abstract}
We discuss the consequences of both electron-phonon and electron-electron couplings in 1D and 2D multi-band (Peierls-Hubbard) models. After briefly discussing various analytic limits, we focus on (Hartree-Fock and exact) numerical studies in the intermediate regime for both couplings, where unusual spin-Peierls as well as long-period, frustrated ground states are found. Doping into such phases or near the phase boundaries can lead to further interesting phenomena such as separation of spin and charge, a dopant-induced phase transition of the global (parent) phase, or real-space ("bipolaronic") pairing. We discuss possible experimentally observable consequences of this rich phase diagram for halogen-bridged, transition metal, linear chain complexes (MX chains) in 1D and the oxide superconductors in 2D.
\end{abstract}

\section{INTRODUCTION}

The MX chain class of materials [1] and oxide high-temperature superconductors (HTS) are striking similar from the perspective of strong competitions for broken-symmetry ground states — bondorder-waves (BOW), charge- (CDW) or spin- (SDW) density-waves or antiferromagnetism (AF), or both bond- and spin- distortions (spin-Peierls, SP) — and the properties of doping- and photo-induced local defect states (kinks, polarons, bipolarons, excitons, breathers, etc.) within the same wide variety of novel ground states. It is becoming increasingly apparent that to correctly model these materials, not only must one take into account both electron-electron (e-e) and electron-phonon (e-p) interactions, but also that multi-band and/or multi-orbital effects are critically important.

Experimentally, the remarkable tunability of the MX class, along with the ease of synthesis of single crystals, is a major advantage allowing systematic investigation between small and large polaron regimes, the influence of dimensionality, and the competition with non-adiabatic and impurity/localization effects. Intrinsic defects (self-trapped local defect states or bags) in both HTS and MX chain materials are thought to be polaronic in nature, and can be created via doping or photoexcitation. Electron-hole asymmetry, which has been proposed as a driving mechanism for superconductivity (SC), has been experimentally observed in MX compounds [2]. Recent evidence in PtBr suggests bipolaron dissociation and polaronic trapping as temperature is varied [3]. Moreover, the model described below 
manifests long-period (LP) ("superlattice") phases [4], possibly observed in recent experiments on MX compounds, and may be related to twinning or real space pairing in the cuprate HTS. Importantly, $\mathrm{SC}$ is believed to occur at the regions of "melting" between various broken symmetry ground states and correlated metals. Thus we anticipate the possibility of SC, or one dimensional (1D) precursors of $\mathrm{SC}$, in MX materials when we are near metal-insulator boundaries, where bipolarons are extended and overlapping, competing with a correlated metal state. Here also the tunability of the MX class can be utilized, leading to our current strategy of studying PtI and $\mathrm{Pd}_{x} \mathrm{Ni}_{1-x} \mathrm{Br}$ to tune into weak CDW and SDW regimes. Recent unusual results on PtI in high magnetic fields [5] may indicate such a 1D precursor.

Theoretically, MX materials are clearly a 1D template [6] for the same many-body methods and parameter determinations one must employ in 2D cuprate and 3D bismuthate HTS models when nonbonding orbitals are neglected. Particularly all are hybridized, multi-band materials. The appearance of superlattice CDW ordering in MX materials [4] may be similar to observations in many HTS materials. In other words including a microscopic driving force for finite scale "twinning" textures and integrating electronic degrees of freedom yield an effective anharmonic lattice dynamics. Indeed, in the model described below the superlattices arise from ordering of "bipolaronic" defects with respect to the period-4 CDW, corresponding to a "melting" of the broken symmetry state ( $\left.c f . \mathrm{Ba}_{1-x} \mathrm{~Pb}_{x} \mathrm{BiO}_{3}\right)$. Polaron pairing into bipolarons is a common ingredient of many current e-e and e-p theories of HTS materials. Further, both $\mathrm{MX}$ chains and (with $\mathrm{M}=\mathrm{Cu}, \mathrm{X}=\mathrm{O}$ ) $\mathrm{CuO}_{2}$ planes (and chains), can be described by essentially the same multi-band, tight-binding extended Peierls-Hubbard Hamiltonian (PHH), described briefly below, although a 3/4-filled, 2-band (2B) 1D model for the MX compounds or a $\mathrm{CuO}$ chain, and a 5/6-filled, 3-band 2D model for the $\mathrm{CuO}$ planes. This same model can be considered a 3/4-filled analog of the the organic conductor polyacetylene, model charge-transfer salts such as TTF-TCNQ, or be used to investigate neutral-ionic transitions [7]. Mathematically, one can consider the two orbitals to be on the same site, and thus this Hamiltonian is also related to the Kondo Hamiltonian used to describe heavy fermion materials.

\section{MULTI-BAND MODEL}

Our model 1D, 2B, PHH focusing on the $\mathrm{d}_{z^{2}}$ and $\mathrm{p}_{z}$ orbitals and including only the nearest neighbor interactions is $[8,9]$

$$
\begin{aligned}
H= & \sum_{l, \sigma}\left\{\left(-t_{0}+\alpha \delta_{l}\right)\left(c_{l, \sigma}^{\dagger} c_{l+1, \sigma}+c_{l+1, \sigma}^{\dagger} c_{l, \sigma}\right)+\left[\epsilon_{l}-\beta_{l}\left(\delta_{l}+\delta_{l-1}\right)\right] c_{l, \sigma}^{\dagger} c_{l, \sigma}\right\} \\
& +\sum_{l}\left\{U_{l} n_{l \uparrow} n_{l \downarrow}+V n_{l} n_{l+1}+\frac{1}{2} K\left(\delta_{l}-a_{0}\right)^{2}\right.
\end{aligned}
$$

where $(\epsilon, \beta, U)_{l}=(\epsilon, \beta, U)_{M, X}$ and $\epsilon_{M}-\epsilon_{X}=2 e_{0}$. For a more detailed description of the parameters, the methods of solution, and general properties of this Hamiltonian, see Ref.s [8] and [9]. The results here were obtained by exact diagonalization (ED) of the many-body PHH on small systems.

Tuning $e_{0}, t_{0}, U_{M}$, and $U_{X}$ is essentially a $1 \mathrm{D}$ analog of the theoretical discussions in $2 \mathrm{D}$ which focus on the $p$ - $d$ hybridization in HTS materials. There, as here, these parameters determine the stoichiometric ground state broken symmetry order, and the nature of electron or hole doping into those ground states. Indeed, the similarity is even stronger because the nominal band filling is essentially the same in both MX and HTS materials - 3/4-filling of 2 bands and 5/6-filling of 3 bands, respectively. Furthermore, both strongly AF and CDW stoichiometric compounds exist for both HTS and MX. We stress the MX class has the advantage of essentially continuous tunability between these extremes. Examining trends in related materials often yields insights that a single material focus might easily 
Fig. 1. The $t_{0}=0$ phase diagram for $\beta_{X} / \beta_{M}=U_{X} / U_{M}=1$. The dashed line is the slice used for Fig. 4 and + the point in Fig. 3. Note changing $P$ translates into moving along a line through the origin.

Table 1. The period-4 phases, occupancies, distortions, energies, and effective $J$ scaling in the $t_{0} \rightarrow 0$ limit. Here $u_{m, x}=U_{M, X} / e_{0}, b_{m, x}=\left(\beta_{M, X}\right)^{2} /\left(K e_{0}\right)$, and $\delta(n)=d_{X}\left(\cos \frac{n \pi}{2}-\sin \frac{n \pi}{2}\right)-d_{M}\left(\cos \frac{n \pi}{2}+\sin \frac{n \pi}{2}\right)$ defines $\mathrm{X}(\mathrm{M})$ sublattice distortion order parameters $d_{X}\left(d_{M}\right)$ with the first bond (X-M) being short. (constant or $(-1)^{n}$ terms just renormalize $t_{0}$ and $e_{0}$ ). For non-period-4 phases see Ref. [4]. For $t_{0} \neq 0$, the unpaired spins are antiferromagneticly correlated.

\begin{tabular}{|c|c|c|c|c|c|}
\hline phase & $X M X M$ & $d_{M}$ & $d_{X}$ & $2 E_{T} /\left(e_{0} N\right)$ & $J^{\mathrm{eff}}$ \\
\hline CDW & $\mathbb{Z}=\bullet=\mathbb{Z}-\mathbb{H}-$ & $\beta_{M} / K$ & 0 & $u_{x}-1+u_{m} / 2-b_{m}$ & - \\
\hline BOW & $\bullet=\mathbb{Z}-\mathbb{Z}-\mathbb{N}=$ & 0 & $\beta_{X} / K$ & $u_{x} / 2-b_{x}+u_{m}+1$ & - \\
\hline MIX & $\uparrow=\downarrow-\mathbb{N} \cdots \mathbb{N}-$ & $\beta_{M} /(2 K)$ & $\beta_{X} /(2 K)$ & $u_{x} / 2-b_{x} / 4+u_{m} / 2-b_{m} / 4$ & $J_{M X} \propto t_{0}^{2}$ \\
\hline MAF & $\mathbb{H}-\uparrow-\mathbb{Z}-\downarrow-$ & 0 & 0 & $u_{x}-1$ & $J_{M M} \propto t_{0}^{4}$ \\
\hline $\mathrm{XAF}$ & $\uparrow-\mathbb{\downarrow}-\downarrow-1-$ & 0 & 0 & $u_{m}+1$ & $J_{X X} \propto t_{0}^{4}$ \\
\hline
\end{tabular}

miss. Finally, we note that competition between e-p and e-e coupling is especially important for for doping states and can induce strong local LD even in a strongly magnetic background $[9,10]$.

\section{ANALYTIC LIMITING CASES}

In the LD CDW case typical of many MX chains, a first approximation is to approach the material as decoupled $\mathrm{X}=\mathrm{M}=\mathrm{X}$ trimers and $\mathrm{M}$ monomers. Indeed, $\mathrm{PtCl}$ chain absorptions are very close to the monomers and trimers in solution. Conversely, when e-e interactions are dominant and the splitting between the bands is large (1B limit), one expects a SP phase in 1D [11], with an effective AF coupling between $\mathrm{M}$ sites given by $J_{M M}=t_{M M}^{2} / U_{M}$, where $t_{M M}$ is the M-M hopping in the effective 1B model. We discuss here the $t_{0}=0$ limit, which captures the essential features of the material in both these limits. Another analytically tractable limit $(U=0)$ was discussed in detail in Ref. [8].

The distortion and energies of the period-4 ( $\mathrm{P} 4)$ phases for $t_{0}=\alpha=0$, listed in Table 1, are: two undistorted configurations with unpaired electrons on the $\mathrm{M}$ (MAF) or X (XAF) sites, one phase with both LD and unpaired spins (MIX) and 2 diamagnetic phases with the M (BOW) or X (CDW) sublattice distorted and a charge-density-wave on the opposite sublattice. The phase diagram for parameters we expect to be relevant to MI or NiX materials, is shown in Fig. 1. The phase diagram is more complex for $U_{X}, \beta_{X} \gtrsim U_{M}, \beta_{M}$, where the hybridization-driven competition is most effective. The BOW (XAF) phase is only found for $\left|\beta_{X} / \beta_{M}\right|\left(U_{X} / U_{M}\right)$ larger than the case shown (though at $t_{0} / e_{0} \sim 2$, or smaller with $\alpha>0$, a BOW phase is found numerically near the MAF phase boundary for these parameters). 


\section{SPIN-PEIERLS AND FRUSTRATED PHASES}

When $t_{0} \equiv 0$, all spin excitations are isoenergetic. For $t_{0} \neq 0$, AF correlations develop and one can treat the problem in terms of an effective spin model [12]. Without coupling between the two bands, the lower, X-like (for $e_{0}>0$ ) band is full and non-magnetic, while the upper (M-like) band is 1/2-full with one electron per $\mathrm{M}$ site (when e-e repulsion is dominant). The on-site e-p coupling $\beta$ leads to a splitting of the upper band which competes with an AF ordering of the spins caused by the effective AF coupling $\left(J_{M M}\right)$ (as in the effective 1B case). When hybridization between the two bands is allowed, the lower band is not completely full, and now there is an effective AF coupling between neighboring halide sites $\left(J_{X X}\right.$, dominant when $U_{X}$ is dominant) as well as $\mathrm{M}$ and $\mathrm{X}$ sites $\left(J_{M X}\right)$, not present in $1 \mathrm{~B}$ models. In fact, when the splitting due to the e-p coupling $\beta$ is on the order of $U$ and $e_{0}$, the AF state with neighboring M-X pairs singly occupied can become the ground state, as shown in Fig. 1. Note this implies, in contrast to the 1B case, that the combination of e-e and e-p coupling in the $2 \mathrm{~B}$ model drives, in addition to the non-magnetic CDW and BOW phases, three (competing) SP phases: one on the X-sublattice (XAF), one on the M-sublattice (MAF), and one involving MX pairs (MIX). Since $J_{M M}$, $J_{X X}$, and $J_{M X}$ are all AF couplings $(J>0)$, they obviously cannot all be simultaneously satisfied and the system is frustrated, as shown in Fig. 2. It is straightforward to derive from fourth-order perturbation theory in the large $U$ limit an effective $t, J_{M X}, J_{M M}, J_{X X}$ model, though the expressions for the $J$ 's are cumbersome and phase dependent [12]. The resultant $t_{0}$ dependencies of the $J$ 's are listed in Table 1 . When $t_{0}$ is small and one is in a regime where only one of the $J$ 's is important, one can numerically check this estimate by comparing the energies of the singlet and triplet ground states (at fixed LD). Fig. 3 shows the $t_{0}$ dependence is correctly predicted. Note the CDW phase has an entirely e-p driven AF component.

In Fig. 4 we show the total energy and average lattice distortion for parameters similar to those used in the $2 \mathrm{D}$ model for $\mathrm{CuO}$ planes [10] (in hole notation, $e_{0}=t_{0} / 2-U_{M} / 4, \beta=0, U_{X} / t_{0}=3, V / t_{0}=1$, $\alpha^{2} / K t_{0}=2$, and the M-M distance was held constant) with analogous results. For small $U_{M}$, the ground state is a CDW, as expected $[8,9]$. As $U_{M}$ increases, the $\mathrm{X}$ no longer symmetrically distort (BOW in notation of Ref. [10]), then a LP phase develops, and finally the SDW phase expected at large $U_{M}$ sets in. In contrast to the $2 \mathrm{D}$ case, the $\mathrm{SP}$ phase is not seen, though a metastable SP regime is found. As is clearly seen from the figure, the "melting" of the CDW phase takes place through a LP intermediary [13]. SC is believed to occur at the regions of "melting" between various broken symmetry ground states and correlated metals. While SC is not expected in the 1D materials, unusual results on PtI in high magnetic fields [5] may be a 1D precursor.

Upon doping, the phase diagram becomes even richer. As has also been seen in the $2 \mathrm{D}$ version of the PHH [10], doping into, e.g., the SDW phase can lead to polaronic defects with local CDW character, and vice-versa. An example of this for our 1D model was reported in Ref. [9] for Ni parameters. Similar results have recently been reported in a 2D model for the HTS [10]. Further, near the phase boundary, we have also found regions where doping into the SDW ground state can cause a CDW defect in a $C D W$ background (and vice-versa) - i.e, the dopant has altered not only it's local environment, but the background phase as well. While clearly finite-size effects will be important for this energy balance, one can easily find parameter regions where the "finite size" is as large as typical correlation lengths in the real materials. Additionally, in the "zero-hopping" limit where the many-body PHH is analytically tractable, one can show that such phase reversal upon doping can occur even in the infinite-system limit. Indeed, the narrow CDW phase seen near 1/8th doping in the La based HTS compounds may be related to this phenomenon.

The crossover from P4 CDW to P4 MAF may also be accompanied by LP superlattice phases [14]. Such superlattice phases have recently been found in a $2 \mathrm{D}, 3 \mathrm{~B}$ model of $\mathrm{HTS} \mathrm{CuO}_{2}$ layers, 
Fig. 2 (left). Schematic energy level diagram in the strongly correlated limit showing frustration of the effective antiferromagnetic couplings caused by MX hybridization.

Fig. 3 (right). The $t_{0}$ dependence of the difference in singlet and triplet energies for the CDW, MIX, and MAF phases at the + in Fig. 1. For small $t_{0}$, this corresponds to excitations of the effective spin-Hamiltonian $H=4 J S_{i} S_{i+j}$ with energy $4 J_{M M}$ in the MAF phase and $4 J_{M X}$ in the MIX phase, whereas in the CDW phase this reflects the Peierls gap.

Fig. 4. (a) Total energy and (b) lattice distortion amplitude as a function of $U_{M}$ for CDW (-), BOW $(--)$, SP (...) SDW (-•), and LP (- -) phases. Parameters consistent with $\mathrm{CuO}$ were used.

including intersite e-p coupling. We anticipate the same behavior in our MX model when intersite e-p coupling is included. Such superlattices may be viewed as an ordered array of discommensuration defects with respect to a nearly stable commensurate order (e.g., P4). In view of the effective J's discussed above, it may be natural to model such states in terms of ANNNI-like models, where nearest and longer range couplings compete, leading to frustration and associated complexity phenomena multitimescale relaxation, hysteresis, metastability, etc. In the context of the MX class, it will be particularly interesting to investigate materials in, or near, this crossover regime - e.g., PtI - and to

further control the crossover with pressure, magnetic field [5], doping, impurities, etc. Indeed doping into this complex regime should be highly sensitive to the softness and competitions of the phases: This may well be an excellent regime to study pairing tendencies and metallization.

\section{CONCLUSIONS}

We stress that the $1 \mathrm{D}, 2 \mathrm{~B}, \mathrm{PHH}$, while simple to write down, is representative of a very large variety of low-D electronic materials. This variety is mirrored in the model's richness, especially in terms of doping near phase boundaries where novel pairing mechanisms are found (inclusion of e-p in a 2D 3B model leads to coexistence of CDW and SDW phases - "charge bags" - as e-p interactions 
are important only in the neighborhood of defects [10]), or a dopant-induced transition [15] of the global phase may exist, besides the usual plethora of doping and photoinduced non-linear excitations. The MX class of compounds are uniquely important as a testing ground for many-body modeling and materials design strategy in strongly correlated, low-D materials (in particular the oxide HTS). Apart from pure and mixed-halide MX materials we are also beginning to explore mixed-metal $\left(\mathrm{M}_{x} M_{1-x}^{\prime} \mathrm{X}\right)$ and bimetallic (MMX) systems, as well as effects of magnetic fields (especially on the weak CDW/SDW ground state materials). We feel that experimental investigations of the pressure dependence and high (magnetic) field behavior of pure and doped materials in the LD/AF cross-over regime, such as PtI, $\mathrm{NiBr}$, or their mixed-metal or -halide analogs, will continue to yield interesting insights into the nature of multiband effects and the competition between e-e and e-p interactions.

Acknowledqements. We acknowledge important discussions with X.Z. Huang, L.A. Worl, R. Dono-

hoe, and B.I. Swanson, J. Shi, among others, and thank E.Y. Loh, Jr. for help in writing the ED code. $\mathrm{ARB}, \mathrm{KY}$, and AS were supported by the US DOE, HR by the Deutsche Forschungsgemeinschaft through SFB 213, and JTG by a NRC-NRaD Research Associateship through a grant from ONR. Supercomputer access was through the ACL at LANL. HR and JTG are grateful to the hospitality of LANL, where this work was begun.

\section{REFERENCES}

1 The structure and chemistry of MX chains, $\left[\mathrm{ML}_{2}\right]\left[\mathrm{ML}_{2} \mathrm{X}_{2}\right] \cdot \mathrm{Y}_{4}$ (M: Pt,Pd,Ni; X: Cl,Br,I; L,Y: various ligands, counterions), has been reviewed by H.J. Keller, in J.S. Miller (ed.), Extended Linear Chain Compounds, Vol. 1, Plenum, New York, 1982, p. 357. For a sampling of the physics literature see, e.g, Proc. of the Int. Conf. on the Sci. and Tech. of Synth. Metals, ICSM '90, Tübingen, Germany, Sept. 2-7, 1990 [Synth. Metals 41-43, (1991)], or Ref.s [8] and [9] and references therein.

2 J.T. Gammel et al., Phys. Rev. B, 42 (1990) 10566.

3 R.J. Donohoe et al., J. Phys. C, (1992), in press.

4 I. Batistić, J.T. Gammel and A.R. Bishop, Phys. Rev. B, 44 (1991) 13228.

5 M. Haruki and P. Wachter, Phys. Rev. B, 43 (1991) 6273.

6 D. Baeriswyl and A.R. Bishop, Physica Scripta, T19 (1987) 239; S.D. Conradson et al., Solid State Commun., 65 (1988) 723; J.T. Gammel et al., Physica B, 163 (1990) 458.

7 A. Painelli and A. Girlando, Synth. Metals 29 (1989) F181.

8 J.T. Gammel et al., Phys. Rev. B, 45 (1992) 6408.

9 S.M. Weber-Milbrodt et al., Phys. Rev. B, 45 (1992) 6435.

$10 \mathrm{~K}$. Yonemitsu et al., to be published in the Proc. of the Int. Conf. on Lattice Effects in High $\mathrm{T}_{c}$ Superconductors, Sante Fe, New Mexico, Jan. 13-15, 1992 (World Scientific).

11 J.E. Hirsch, Phys. Rev. Lett., 51 (1983) 296.

12 J. Shi, R. Bruinsma, and A.R. Bishop, preprint 1992.

13 There are many metastable LP phases, and the LP energies should be treated as upper bounds. From Fig. 4 there is clearly a region where the LP phase is favored over any period- 4 phase. This intermediate LP region is seen in ED, MFA, and the 2D model. It is not an artifact.

14 Intermediate phases can be driven by the competition between $t_{0}, \beta, \alpha$, long-range Coulomb fields, and/or the effective short range $J$ 's. Such intermediate phases can be P4, as expected from commensurability effects, or LP. Note that the large- $\beta$ LP phase discussed in Ref. [4], though related, is somewhat different than the frustration driven, magnetic LP phase discussed here or, in 2D, in Ref. [10] (for large $\alpha$ ). Similar to the ANNNI model, no explicit long range terms are needed as frustration drives effective long range interactions,

15 J. Reichl, unpublished and S. Marianer, unpublished. 